\title{
Improving patient adherence: the last obstacle to achieving hypertension control
}

\author{
Shikai Yu' ${ }^{1}$ Yi Zhang ${ }^{1}$
}

Received: 1 February 2021 / Revised: 6 February 2021 / Accepted: 7 February 2021 / Published online: 24 March 2021

(C) The Japanese Society of Hypertension 2021

Hypertension is a major cardiovascular risk factor, and the control of hypertension can significantly prevent organ damage and further reduce the morbidity and mortality of cardiovascular diseases. However, it seems that there is always a substantial gap between knowing and doing. Although a great deal of effort has been exerted to address hypertension in recent years, the control of hypertension is still relatively poor worldwide, especially in low- to middleincome countries. For instance, the rate of control of hypertension in China was reported to be $37.5 \%$ [1]. Recently, the most updated data based on a national survey in the United States reported the alarming fact that the rate of control of hypertension dropped significantly in 2017-2018 in the United States [2], a country that has done relatively well at reducing hypertension.

There are many factors underlying this situation, but adherence to antihypertensive drugs is one of the major contributors. Data indicated that more than one-third of patients prescribed an antihypertensive drug stopped their initial treatment after 6 months, while less than one-half stopped at one year [3]. Unsurprisingly, poor adherence has been found to be significantly associated with worse clinical outcomes [4, 5]. Therefore, improving antihypertensive drug adherence is unquestionably one of the most important measures in current hypertension management. In clinical practice, physicians often attribute poor adherence solely to the patients themselves. However, studies of medication adherence have indicated that adherence is a complex, multidimensional parameter. According to the framework proposed by the WHO [6], there are five dimensions of the mechanisms underlying suboptimal drug adherence, namely, socioeconomic, healthcare system-related, condition-related, therapy-related and patient-related factors.

Yi Zhang

yizshen@gmail.com

1 The Department of Cardiology, Shanghai Tenth People's Hospital, Tongji University School of Medicine, Shanghai, China
This framework, to some extent, has shaped the subsequent research on medication adherence.

In this issue of the journal, Vallée et al. [7] specifically investigated the impact of socioeconomic factors and primary care medicine on treatment and adherence to antihypertensive drugs by analyzing the data derived from the Esteban survey, which was designed to be a representative sample of French adults. Using the medical prescription data extracted from the national electronic health database, the authors estimated adherence to antihypertensive medication as the proportion of days covered (PDC) by the antihypertensive drug treatment during the observational period. This method is widely used in large-sample studies [8], although there is no standardized estimation method. Among 2105 participants with valid BP measurements out of 3031 subjects in the Esteban cohort, 396 patients aware of their hypertension diagnosis were finally included in the study. Of those patients, $66.2 \%$ were treated and $40.9 \%$ were adherent to treatment with antihypertensive drugs. Consistent with other studies [9], Vallée et al. showed that both the rates of treatment and adherence to medical treatment in France are less than satisfactory, suggesting that suboptimal adherence is also a current challenge in France.

Although many socioeconomic factors were included in the analysis, the authors found that only three factors (male sex, diabetes and living with a partner) were significantly associated with antihypertensive drug adherence, whereas age, income and education level were not associated with antihypertensive medication adherence. Currently, there is no final verdict on the associations between these socioeconomic factors and drug adherence, because not all these factors are consistently associated with medication adherence across all studies. In Vallée et al.'s study, the absence of an association between income level and adherence is not surprising, given that France is a developed country with a health insurance system that covers all antihypertensive medication costs. However, this is obviously not the case in low- to middle-income countries [10]. The number of antihypertensive drugs taken by patients has been shown to be significantly associated with adherence to 
medication [11]. However, interestingly, no association was observed between the number of antihypertensive drugs and PDC in Vallée et al.'s study. This might be, at least in part, due to the relatively small sample size of the study.

One unexpected finding in Vallée et al.'s study was that general practitioner (GP) visits and consultations were not associated with drug adherence. In theory, GPs are the medical professionals primarily involved in the routine care of patients with hypertension and supposedly play an important role in improving medication adherence. On the one hand, this absence of an association could be caused by the lack of power in the study because of the small sample size. On the other hand, this implies that some factors (burn-out, problematic doctor-patient relationship, etc.) may prevent GPs from performing their job adequately. From this point of view, more investigations are needed to explore the factors influencing the association between GPs and drug adherence, and more work may be needed to enhance the role played by GPs in hypertension management in the future.

Since the first diagnosis of hypertension, tremendous progress in the recognition and management of this disease has been made, including but not limited to the development of effective therapeutic strategies and medication. However, as Dr. Charles Everett Koop said, "Drugs don't work in patients who don't take them". For hypertension control to be achieved, improving adherence is the last major factor that must be addressed.

\section{Compliance with ethical standards}

Conflict of interest The authors declare no competing interests.

Publisher's note Springer Nature remains neutral with regard to jurisdictional claims in published maps and institutional affiliations.

\section{References}

1. Wang Z, Chen Z, Zhang L, Wang X, Hao G, Zhang Z, et al. Status of hypertension in china: results from the china hypertension survey, 2012-2015. Circulation. 2018;137:2344-56.

2. Muntner P, Hardy ST, Fine LJ, Jaeger BC, Wozniak G, Levitan EB, et al. Trends in blood pressure control among us adults with hypertension, 1999-2000 to 2017-2018. JAMA. 2020;324:1190-200.

3. Vrijens B, Vincze G, Kristanto P, Urquhart J, Burnier M. Adherence to prescribed antihypertensive drug treatments: Longitudinal study of electronically compiled dosing histories. BMJ. 2008;336:1114-7.

4. Herttua K, Tabák AG, Martikainen P, Vahtera J, Kivimäki M. Adherence to antihypertensive therapy prior to the first presentation of stroke in hypertensive adults: Population-based study. Eur Heart J. 2013;34:2933-9.

5. Shin S, Song H, Oh S-K, Choi KE, Kim H, Jang S. Effect of antihypertensive medication adherence on hospitalization for cardiovascular disease and mortality in hypertensive patients. Hypertens Res. 2013;36:1000-5.

6. World health organization. Adherence to long term therapies: evidence for action. Geneva: World health organization; 2003.

7. Vallée A, Grave C, Gabet A, Blacher J, Olié V. Treatment and adherence to antihypertensive therapy in france: the roles of socioeconomic factors and primary care medicine in the esteban survey. Hypertens Res. 2021. https://doi.org/10.1038/s41440-02000603-z. Epub ahead of print.

8. Nishimura S, Kumamaru H, Shoji S, Sawano M, Kohsaka S, Miyata $H$. Adherence to antihypertensive medication and its predictors among non-elderly adults in japan. Hypertens Res. 2020;43:705-14.

9. Mennini FS, Marcellusi A, von der Schulenburg JM, Gray A, Levy P, Sciattella $\mathrm{P}$, et al. Cost of poor adherence to anti-hypertensive therapy in five european countries. Eur J Health Econ. 2015;16:65-72.

10. Macquart de Terline D, Kane A, Kramoh KE, Ali Toure I, Mipinda JB, Diop IB, et al. Factors associated with poor adherence to medication among hypertensive patients in twelve low and middle income sub-saharan countries. Plos ONE. 2019;14:e0219266.

11. Ishida T, Oh A, Hiroi S, Shimasaki Y, Nishigaki N, Tsuchihashi $\mathrm{T}$. Treatment patterns and adherence to antihypertensive combination therapies in japan using a claims database. Hypertens Res. 2019;42:249-56. 